\title{
Cosmic crystallography using short-lived objects - Active Galactic Nuclei
}

\author{
A. Marecki ${ }^{1}$, B. F. Roukema ${ }^{1}$, and S. Bajtlik ${ }^{2}$ \\ 1 Toruń Centre for Astronomy, N. Copernicus University, ul. Gagarina 11, 87-100 Toruń, Poland \\ e-mail: amr@astro.uni.torun.pl \\ 2 Copernicus Astronomy Centre, ul. Bartycka 18, 00-716 Warsaw, Poland
}

Received 6 October 2004 / Accepted 5 January 2005

\begin{abstract}
Cosmic crystallography is based on the principle that peaks in the pair separation histogram (PSH) of objects in a catalogue should be induced by the high number of topologically lensed pairs that are separated by Clifford translations, in excess to "random" pairs of objects. Here we present modifications of this method that successively improve the signal-to-noise ratio by removing a large part of the noise and then false signals induced by selection effects. Given the transient nature of the most readily available tracer objects, Active Galactic Nuclei (AGNs), the former is possible because a natural filter for removing many of the noise pairs is available: when counting pairs of objects in order to create PSHs, only those with nearly identical redshifts need to be counted. This redshift filter (a maximum value of $\Delta z / z=0.005$ ) was applied to a compilation of AGN catalogues. Further noise was removed by applying a second filter, a maximum angle $\Delta \theta=0.075 \mathrm{rad}$, and a minimum number of pairs $n_{\text {pairs }}=3$ to find each "bunch of pairs" $(\mathrm{BoP})$ where the vectors (in Euclidean comoving space) defined by pairs are required to be nearly equal, whereas in the PSH only the lengths must be nearly equal. These filters reveal significant signals, which, however, are due to selection effects. A third filter, a minimum length $L_{\text {selec }}=150 \mathrm{~h}^{-1} \mathrm{Mpc}$ between the (parallel) vectors in a BoP, is found to effectively remove these selection effect pairs. After application of these successive filters, no significant topological signal was found.
\end{abstract}

Key words. cosmology: observations - cosmological parameters - galaxies: quasars: general

\section{Introduction}

General relativity (GR) relates the local geometry and content of space-time in the limit as one approaches any given point. Together with assumptions about approximate local homogeneity and local isotropy ${ }^{1}$, the equations of GR relate the metric properties of space (curvature) and the average material contents and dynamics (density, expansion rate, cosmological constant) of the Universe.

GR is, however, only a local theory that does not constrain global geometry: the curvature of space does not tell us whether the Universe is finite or infinite in spatial volume (or mass). Schwarzschild $(1900,1998)$ pointed out a century ago that a flat, homogeneous universe can be finite if the topology of space is not trivial, i.e. if space is multiply connected. De Sitter (1917) and Friedman (1923) also noted this, while Friedman (1924) pointed out that even a universe of negative curvature could be homogeneous but finite. In contrast to local geometry, which is constrained by GR, there is neither consensus on a physical theory that would predict what the topology of the Universe should be nor any theory that would relate

${ }^{1}$ For comments on "local" versus "global" homogeneity and isotropy, see Roukema (2002a). that topology to dynamical properties of the matter distribution, even though work on some elements of what might be needed has begun, e.g. Dowker \& Surya (1998); Hawking (1984a,b); Zeldovich \& Grishchuk (1984).

Nearly all methods for detecting the topology of the Universe rely on the principle of multiple topological imaging of either discrete objects or of the thermally radiating plasma seen in the cosmic microwave background radiation. This phenomenon is called "topological lensing". Detection of topological lensing would allow us to directly study the evolution of individual objects over a cosmological time-scale, as the same object would be visible at different cosmic epochs.

Although the amount and the quality of extragalactic observations available is growing constantly, and many different observational strategies are possible, detection of topological lensing has so far proved to be very difficult. Reviews of different strategies include, e.g. Uzan et al. (1999a); Luminet \& Roukema (1999); Roukema (2002b); Rebouças \& Gomero (2004).

One of the methods, "cosmic crystallography" (Lachièze-Rey \& Luminet 1995; Lehoucq et al. 1996), was explained and applied to some catalogues of observational data, but no significant detection has so far been obtained. 
Is further application of this method still worth trying? Arguments in favour include:

(i) the microwave background observations of DASI, BOOMERanG, MAXIMA, DMR and CBI lead to $\Omega_{\text {tot }} \simeq 1$ (Sievers et al. 2003). This estimate has been strongly underpinned by the WMAP-based result: $\Omega_{\text {tot }}=1.02 \pm 0.02$ (Spergel et al. 2003). The fact that the Universe inside of the observable horizon appears to be nearly flat makes cosmic crystallography a viable method;

(ii) Active Galactic Nuclei (AGNs) have short lifetimes as active (easily visible) objects, leading to (approximately) removable noise in the pair histograms. In this work, a method of removing these pairs and improving the signalto-noise ratio is introduced.

The main argument against applying cosmic crystallography is that recent attempts at constraints using the WMAP microwave background data have so far failed to detect matched circles for torus-like models (e.g. Cornish et al. 2004). Also, analyses of the WMAP data are accumulating that suggest that the global topology of the Universe may be that of the Poincare dodecahedral space (Luminet et al. 2003; Roukema et al. 2004). Clearly, at any time well past the quantum epoch, $t \gg 10^{-43} \mathrm{~s}$, the comoving spatial section of the Universe cannot simultaneously be both a Poincaré dodecahedral space and a toroidal 3-manifold.

These reasons are not, however, sufficient for choosing not to develop and apply several tests for such a fundamental property of the Universe by independent methods, using independent sets of objects. The cosmic microwave background (CMB) remains difficult to interpret and filter for foreground contamination and for the integrated Sachs-Wolfe Effect, and even the internal linear combination WMAP map (ILC) is found to include significant foreground components at low $l$ values according to some authors (Naselsky et al. 2003, 2004). Moreover, even if individual pixels in CMB data, rather than statistical properties, were perfectly resolved into their respective cosmological components, it would still be useful to have physically independent checks on CMB results.

In this article, the method of reducing the noise in the cosmic crystallography method - in the case of a catalogue of AGNs - is presented in Sect. 2. This involves successive filters, parametrised by:

(i) $\Delta z$ - a maximum redshift difference criterion to remove many of the noise pairs in a pair catalogue;

(ii) $\Delta \theta, n_{\text {pairs }}$ - to remove pairs which are least likely to form "spikes" of topological origin;

(iii) $L_{\text {selec }}$ - to remove selection effect spikes which imitate topological spikes.

Results are presented in Sect. 3.2 and are summarised in Sect. 4. Notation adopted here for local cosmological parameters includes $\Omega_{\mathrm{m}}$ (present matter density), $\Omega_{\Lambda}$ (cosmological constant and/or quintessence constant), $w$ (quintessence parameter), and the Hubble constant parametrised as $h \equiv$ $H_{0} / 100 \mathrm{~km} \mathrm{~s}^{-1} \mathrm{Mpc}^{-1}$.

\section{The method}

\subsection{The cosmic crystallography method and its variations}

The cosmic crystallography method was originally proposed by Lachièze-Rey \& Luminet (1995) and presented in more detail in Lehoucq et al. (1996). In brief, the idea was the following. If the Universe (more specifically: the comoving spatial section of the Universe) is multiply connected, then each image of a given object is linked to each of the other images of the object by the holonomies of the space. Without knowing the curvature or the topology we do not know what these holonomies are, but we know that they are isometries. Therefore, any pair of images of a single object can be related by an isometry. At the separation distance corresponding to such an isometry, the probability of finding two objects separated by that distance should be higher than in the absence of the isometry. In other words, the spatial two-point auto-correlation function of a catalogue of objects should show excess correlations at certain pair separations.

Lehoucq et al. (1996) presented this as a non-normalised auto-correlation function, simply plotting counts of pairs of objects and calling the function a "pair separation histogram" (PSH). They carried out several simulations starting from an artificial catalogue that consisted of a moderate number of objects randomly distributed in a fundamental polyhedron with equal edge lengths. In such an idealised case, the excess correlations that gather pairs linked by holonomies stand out dramatically above the background distribution of other pairs, and so are termed "spikes". Lehoucq et al. (1996) pointed out that when applied to real data (they used a catalogue of galaxy clusters), many factors start to play a destructive rôle, i.e. by smearing out such sharp spikes, which makes signatures of the possible existence of ghost images difficult to detect. Uzan et al. (1999a) went on to apply the PSH test to a quasar catalogue and found no topological signal.

An alternative proposal for collecting "generator pairs" (or "type II pairs"), as in cosmic crystallography, was the the search for local isometries, i.e. to group together "local pairs" (or "type I pairs"), or more generally, local $n$-tuplets (Roukema 1996). In Uzan et al. (1999b), the special case of $n$-tuplets with $n=2$, i.e. pairs, was discussed and extended.

Uzan et al. (1999b) extended the 2-tuplet case to provide a single statistic, equivalent to the auto-correlation function of the spatial two-point auto-correlation function, and named this the "collecting-correlated-pair" (CCP) statistic, which should have a high value in the presence of multiple topological imaging in a population composed of good standard candles, whether space is hyperbolic, flat, or spherical. Since there should only be a significant signal for the correct values of the curvature parameters $\Omega_{\mathrm{m}}$ (present matter density), $\Omega_{\Lambda}$ (cosmological constant and/or quintessence constant), and $w$ (quintessence parameter), a signal could potentially be detected as a relative signal without having to calculate its absolute significance.

Application of the CCP method to a quasar catalogue again gave no topological signature. The authors were limited by computer time in spanning the full parameter space with high 
resolution in parameter values, but the principle is certainly valid.

Another way to apply a similar test is for the case where: (1) the curvature parameters' values are known; (2) the Universe is negatively curved; and (3) a particular hypothesis is made regarding the 3-manifold of space. This is known as the "pullback method" (Fagundes \& Gausmann 1999).

While the PSH test is designed to detect topological lensing, it has been pointed out by Gomero et al. (2002) that (1) it is likely to miss some part of the real signal of topologically lensed pairs; and (2) it would be insufficient to determine which 3-manifold we live in:

(1) the spikes of topological origin in single PSH's are only due to translations of the covering group, whereas correlations due to the other (non-translational) isometries manifest as small deformations of the PSH of the universal covering space. Hence, some part of the real signal would be missed (if a rotation in the covering space is needed when matching faces);

(2) distinct Euclidean manifolds that admit the same translations of their covering space would present the same spike spectrum, despite being different; therefore, the set of topological spikes in the PSHs is not sufficient for distinguishing these compact flat manifolds, making it clear that even if the comoving spatial section of Universe is globally flat and observably multiply connected, the spike spectrum may not be enough for determining its global shape.

Another way of stating (1) is that among the locally homogeneous, flat 3-manifolds, the PSH method works for the 3-torus, because the distance from a source to its nearest image does not depend on its location, but for all other locally homogeneous, flat 3-manifolds, the PSH method detects only a subgroup of the full holonomy group, i.e. the subgroup of pure translations (Weeks et al. 2003).

However, neither of these limitations suggest that the method should not be used; any signal that appeared to be significant would imply a family of hypotheses to be further tested (depending on telescope time allocation).

\subsection{Modifications to the cosmic crystallography method}

Although the active phase of a typical galaxy is believed to be a recurrent phenomenon rather than happening just once in its lifetime (see e.g. Hatziminaoglou et al. 2001), the fraction of time during which a galaxy is in an active phase is likely to be small. For quasars, for which the comoving space number density at $z \sim 1-2$ is about a thousand times less than that of galaxies, the fraction of time during which they are active is likely to be similar, about $10^{-3}$. This makes the chance of seeing both members of a pair of topological images small, though not zero. Neglecting the "ephemeric" nature of quasars is the factor that may have led Uzan et al. (1999a,b) to have missed a true signal in the catalogue they analysed.

\subsubsection{Maximum redshift difference: $\Delta z$}

The short lifetime of AGNs implies that any pair of topological images of a single AGN is most likely to be seen during a single period of activity of the AGN, i.e. during a short time or redshift interval, $\Delta z$. From the light travel time argument and assuming that the length of an activity period is of the order of $10^{7}$ years, we adopted $\Delta z / z=0.005$ as a reasonable amount for a wide range of redshifts.

By removing all pairs of objects at $z_{i}, z_{j}$ for which $\left|z_{i}-z_{j}\right| / z_{i}>\Delta z / z$, the number of potential topological image pairs in the catalogue will be unchanged, while the total number of pairs will decrease by a large factor. Hence, the signalto-noise ratio should increase noticeably.

\subsubsection{Bunches of pairs (BoPs): minimum number of pairs $n_{\text {pairs }}$ within maximum angular difference $\Delta \theta$}

Moreover, if the pairs are due to translations in Euclidean comoving space, then the vectors drawn between the two members of any pair should be parallel to one another. Therefore, a second filter can be applied, namely, selecting those sets of pairs where at least $n_{\text {pairs }}$ vectors connecting those pairs point in (nearly) the same direction. Such a collection is referred to as a "bunch of pairs" (BoP). This means that any lone pair of objects pointing to a given direction, isolated from other pairs but fulfilling the redshift similarity criterion, is removed by this filter, so it is not taken into account at all.

In this analysis, $n_{\text {pairs }}=3$ is adopted; i.e. if in a given direction (within some angular tolerance - see below) there are only two pairs of objects fulfilling the redshift similarity criterion and have vectors in this direction, then these pairs are treated as coincidences and disregarded. In practice, it turned out that the adoption of $n_{\text {pairs }}=3$ is very conservative, since even BoPs with $n_{\text {pairs }}=5$ constitute "noise".

According to this definition, therefore, a BoP consists of at least 3 vectors connecting pairs of AGNs, and these vectors must fulfill the condition that the angle between any two of them is $\langle\Delta \theta$, where $\Delta \theta$ is a small angle.

A physically reasonable value of $\Delta \theta$ depends on possible errors in the three-dimensional spatial positions of the two AGNs contributing to a pair. As mentioned in Roukema (1996), an upper limit of a few $100 \mathrm{~km} \mathrm{~s}^{-1}$ on peculiar velocities limits errors in positions to about $1-10 h^{-1} \mathrm{Mpc}$. For pair separations of about 3000-6000 $\mathrm{h}^{-1} \mathrm{Mpc}$, this implies maximum errors of about 0.003 radians. However, to be conservative, in order not to remove too many pairs that might correspond to a true signal, a much larger tolerance can be adopted.

In general, particularly given the non-uniform distribution of the objects with known redshifts in the catalogue we used, varying $\Delta \theta$ has a clear effect on the distribution of BoPs. If a high value of $\Delta \theta$ is set, several BoPs are merged together, or "smeared", while for lower values, both the "noise" and a potential signal are suppressed. The smearing effect can be monitored by checking the distribution of the angles of vectors within BoPs. For the present study, a $\Delta \theta$ which is low enough to avoid the "smearing" effect of BoPs was found by starting with 


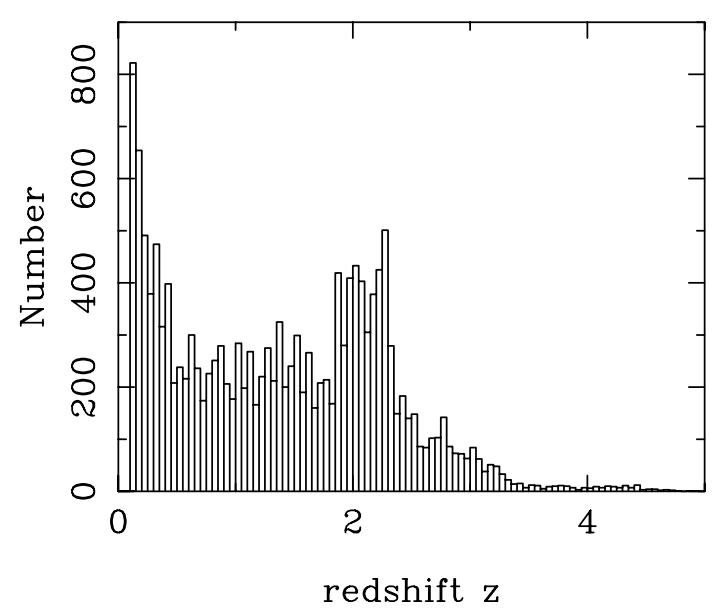

Fig. 1. Redshift distribution of the sample used for analysis - see Sect. 3.1.

a high value and decreasing it until the "poorly collimated" BoPs vanished. This value, $\Delta \theta=0.075 \mathrm{rad}$, is (conservatively) well above the limit to which angular errors are likely to occur due to observational effects such as peculiar velocity and the precision of redshift estimates.

\subsubsection{Selection effects and how to avoid them: $L_{\text {selec }}$}

If a topological signal is present in an AGN catalogue, then a PSH filtered for short lifetimes and confined to the BoPs should show a higher signal-to-noise spike at the length of one of the generators of the 3-manifold of space than in the absence of these filters. However, the presently available AGN catalogues generally include deep and narrow surveys in which many objects are located in a small solid angle or else in a long but narrow angular strip of the $\mathrm{sky}^{2}$. It is obvious that a pair of (small) patches of the sky well-sampled with observations increases the probability that there will be objects of the same redshift in each patch and so would mimic a topological signal in a PSH. Effects of clustering are likely to generate higher than random numbers of parallel vectors, of nearly equal length, between pairs of objects. This is a selection effect that is likely to lead to non-topological spikes in a PSH.

A third filter is possible to counter this effect. While spikes resulting from this selection effect are caused by objects clustered in small regions of space, spikes that represent topological pairs should occur for objects spread throughout space. So, by plotting the vectors represented in a spike in combination with the spatial positions of the objects from which they originate, it should be possible to judge whether the spike is caused by a selection effect or whether it is consistent with topological lensing. More quantitatively, a minimum spatial separation $L_{\text {selec }}$ between the objects generating the pairs in a bunch can be defined in order to exclude most of the pairs from any BoP generated by this selection effect. Any spike of topological origin should remain after the application of the $L_{\text {selec }}$ criterion,

\footnotetext{
${ }^{2}$ We deliberately ignore the SDSS for this very reason - see Sects. 3.1 and 4.
}
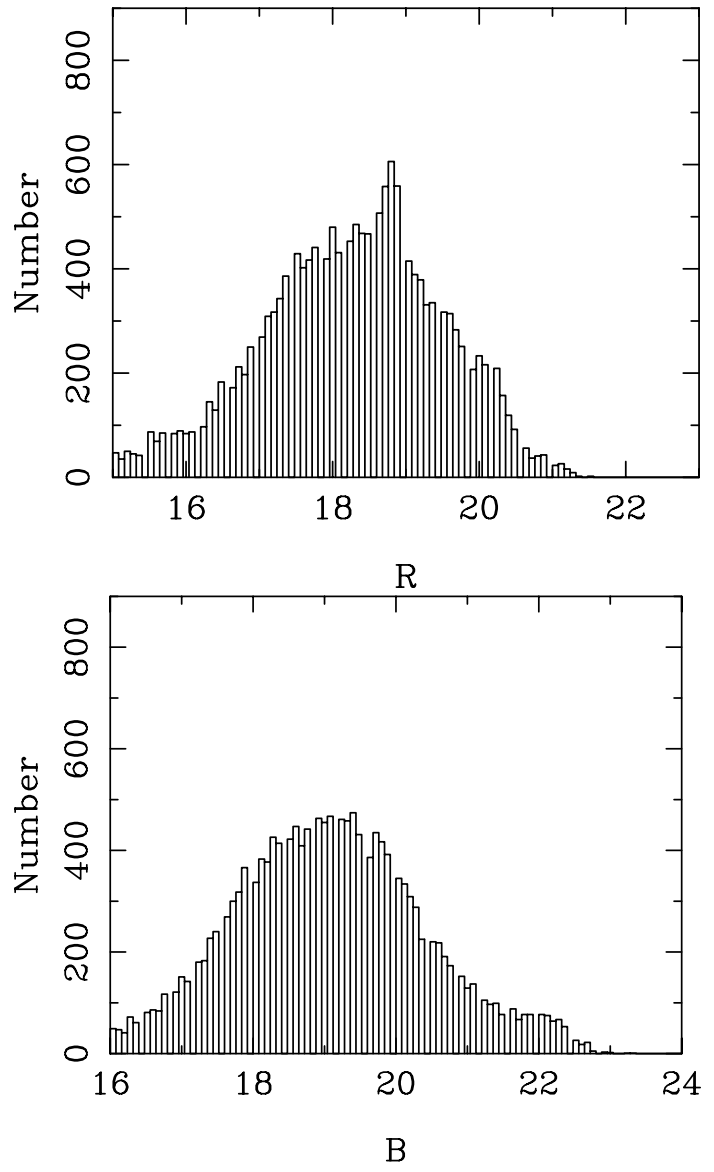

Fig. 2. Apparent $R$ (upper panel) and $B$ (lower panel) magnitude distribution of the sample used for analysis - see Sect. 3.1.

while artefactual spikes, caused by this selection effect, should be removed.

\section{Results}

\subsection{The sample of AGNs}

We used an early version of the catalogue compiled by Flesch \& Hardcastle $(2004)^{3}$ which aligns and overlays the year 2000 releases of the ROSAT, HRI, RASS, PSPC, and WGA $\mathrm{X}$-ray catalogues, the NVSS and FIRST radio catalogues, the Veron QSO catalogue, the Principal Galaxy Catalogue, and the SDSS-EDR onto the optical APM and USNO catalogues. The version we used is dated 18 October 2001. It contains 26533 objects with known redshifts, but we actually took 15762 with $z>0.1$ for our analysis. The redshift and apparent magnitude distributions of this sample of 15762 objects are shown in Figs. 1 and 2.

The dominant observational uncertainties that are relevant for pair separation histogram analysis are the redshifts. Angular positions on the sky are known for these objects to better than an arcsecond, which corresponds to an error of much less than a megaparsec in the sky plane.

Typical redshift uncertainties are \pm 0.01 in the redshift value, corresponding to measurement errors of about

\footnotetext{
3 Available at ftp://ftp. quasars.org
} 
$30 h^{-1} \mathrm{Mpc}$ at $z=0.10$ or about $10 h^{-1} \mathrm{Mpc}$ at $z=3.00$. Additional uncertainty in the true distance estimates for a fixed set of local cosmological parameters $\left(\Omega_{\mathrm{m}}, \Omega_{\Lambda}, w, H_{0}\right)$ comes from the unknown peculiar velocities of the objects relative to the comoving coordinate frame. A peculiar velocity of a few $100 \mathrm{~km} \mathrm{~s}^{-1}$ would give about $1-10 h^{-1} \mathrm{Mpc}$ additional radial uncertainty in position.

We also attempted to explore a newer version of that catalogue which includes the Sloan Digital Sky Survey/Data Release 1 (SDSS/DR1) (Schneider et al. 2002). It turned out, however, that this introduced a large selection effect, as discussed in Sect. 2.2.3 which had to be removed anyway. Consequently, we abstained from trying any of the more recent but - at the time of writing - still incomplete releases of SDSS.

\subsection{Application of the method}

\subsubsection{Maximum redshift difference: $\Delta z$}

As discussed in Sect. 2.2, a tight constraint has to be imposed on $\Delta z$ in order to remove most pairs that do not represent an AGN in a single active phase, while also including some tolerance to reflect observational uncertainty in three-dimensional comoving spatial positions caused by peculiar velocities and inaccuracies in measured AGN redshifts. Figure 3 shows the PSH for all objects we used for our analysis (Sect. 3.1) assuming local cosmological parameters $\Omega_{\mathrm{m}}=0.3, \Omega_{\Lambda}=0.7, w=-1$ after filtering with $\Delta z / z=0.005$.

No obvious signal is present in this figure; despite removing a large part of the noise, either no signal is present at all, or any signal present is still hidden. As the following discussion shows, non-topological signals are present but require further filtering to successively detect and then remove them.

\subsubsection{Bunches of pairs (BoPs): Minimum number of pairs $n_{\text {pairs }}$ within maximum angular difference $\Delta \theta$}

As described in Sect. 2.2.2, pairs caused by topological generators should be parallel to each other. Figure 4 shows the result of following the $\Delta z / z=0.005$ criterion by looking for BoPs with $\Delta \theta=0.075 \mathrm{rad}$ and $n_{\text {pairs }}=3$.

As can been seen in Fig. 4 the concept of BoPs works quite well as a filter of the "noise" introduced by pairs of nontopological origin: several spikes with good signal-to-noise ratios clearly become visible when in addition to taking into account AGNs' short lifetimes - an effect of the $\Delta z$ filter - we also reject all the pairs that do not constitute BoPs with at least three members $\left(n_{\text {pairs }}=3\right.$ ).

While the signal-to-noise has again been improved, this is still insufficient to establish the topological nature of the signal. In particular, the apparently good signal may be the result of selection effects. Clearly, at least some of those spikes are likely to be non-topological in origin, since there is a distribution of many spikes close together, especially for the spikes at around 2-3 $h^{-1} \mathrm{Gpc}$, whereas the error in distances for a true spike should not be larger than about $10 h^{-1} \mathrm{Mpc}$. If any of the spikes

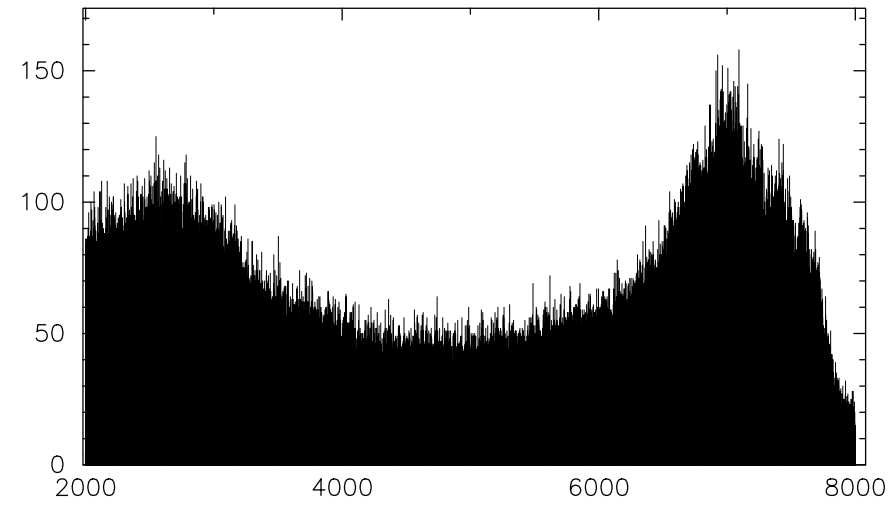

Fig. 3. PSH (pair separation histogram) for the objects in our sample. The only filter applied is requiring the redshifts to both objects in a pair to be very similar - Sect. 2.2.1. The error tolerance in the determination of redshifts is $0.5 \%$ difference in redshifts in a pair. The horizontal axis shows separations of objects in pairs in units of $h^{-1} \mathrm{Mpc}$. The vertical axis shows the number of pairs in a separation bin equal to $1 h^{-1} \mathrm{Mpc}$.

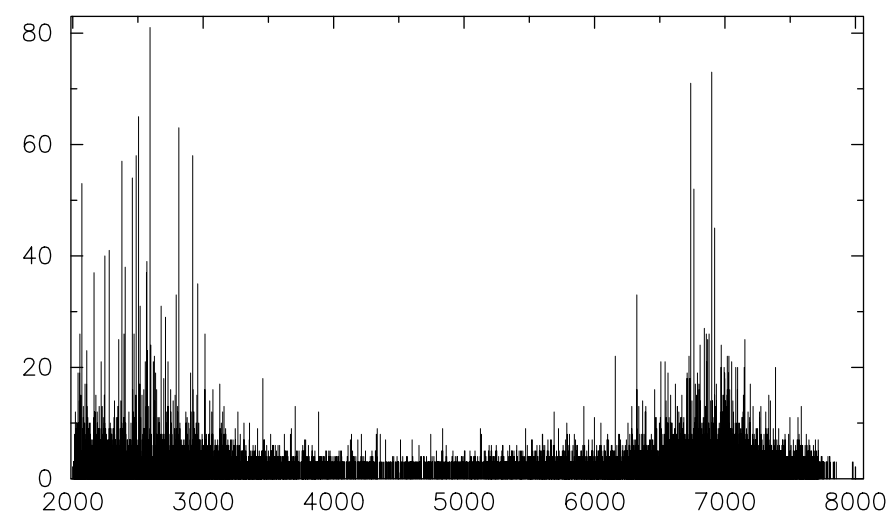

Fig. 4. PSH of Fig. 3 modified according to the BoP filter (Sect. 2.2.2). The horizontal axis shows separations of objects in pairs in $h^{-1} \mathrm{Mpc}$. The vertical axis shows the number of nearly parallel pairs in a bunch. As in the case shown in Fig. 3 we allowed for $0.5 \%$ difference of redshifts in each pair. The angular separations of pairs in a bunch of pairs is $\Delta \theta<0.075 \mathrm{rad}$, and $n_{\text {pairs }}=3$.

are topological, then they just happen to have a signal of about the same amplitude as noise (or selection effect) spikes.

\subsubsection{Selection effects and how to avoid them: $L_{\text {selec }}$}

Before applying the $L_{\text {selec }}$ criterion to remove selection effect spikes (Sect. 2.2.3), it is interesting to look at the orientation of all the BoPs of Fig. 4, which is plotted in Fig. 5. Since the peaks happen to emerge in two groups $\left(2-3 h^{-1} \mathrm{Gpc}\right.$ and $6-8 h^{-1} \mathrm{Gpc}$ ), two plots are presented, one for each of these groups. It turns out that the bifurcation we observe in Fig. 4 reflects the bias caused by the Galaxy, i.e. the scarcity of extragalactic observations near the galactic equator (the so-called zone of avoidance). The BoPs present in the upper panel of Fig. 5, where the distances in pairs are $<5 h^{-1} \mathrm{Gpc}$ (the "short" ones), connect objects mostly on the same side of the galactic equator, either north or south. Therefore, the "short" BoPs predominantly point towards low galactic latitudes. 

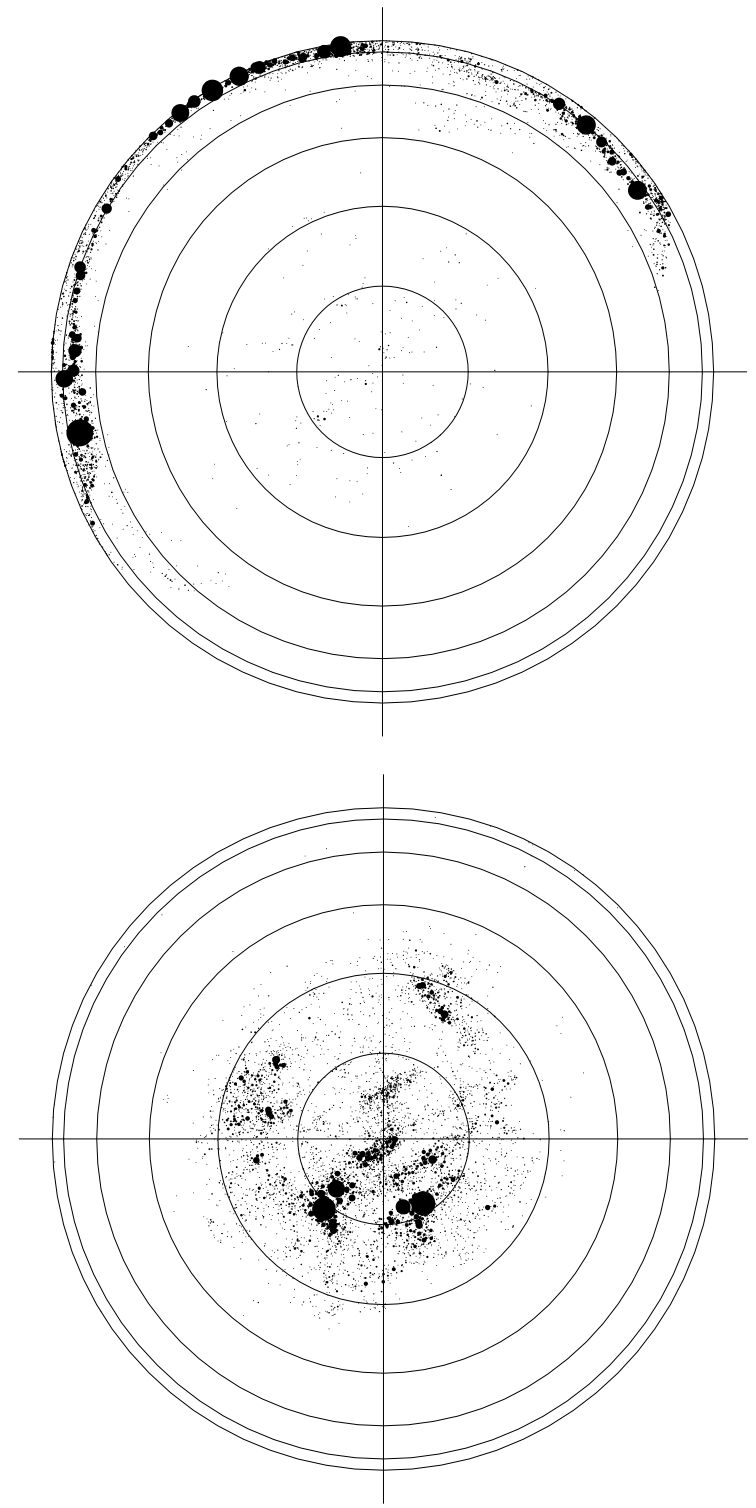

Fig. 5. The distribution of directions the BoPs (Sect. 2.2.2) point to in galactic coordinates. The plots are in polar coordinates: the north galactic pole is in the centre, circles are plotted for $b=75^{\circ}, 60^{\circ}, 45^{\circ}, 30^{\circ}, 15^{\circ}, 0^{\circ}$. The upper panel is for pairs shorter than $5 h^{-1} \mathrm{Gpc}$; the lower panel is for pairs longer than $5 h^{-1} \mathrm{Gpc}$. The radii of filled circles marking the positions are proportional to the number of pairs in a particular BoP.

On the other hand, the BoPs present in the lower panel connect the objects on both sides of the galactic equator, and so they largely point to high galactic latitudes. This explains why the histogram is bimodally distributed, but the question of whether any spikes are topological remains.

The most outstanding spikes in Fig. 4 are the two at about $7000 \mathrm{~h}^{-1} \mathrm{Mpc}$. However, as mentioned in Sect. 2.2.3, what we could and should expect is an ubiquity of false signals resulting from an uneven distribution of objects with known redshifts, since spectroscopic observations are often concentrated in small solid angles.

A visual way to investigate this for a particular peak in the histogram of BoPs is to plot the distribution of pairs in a given
BoP in the cartesian coordinate system where the $z$ axis points to the averaged direction that the BoP points to. If a given BoP is a result of multiple connectedness, a global property of the Universe, then the distribution of pairs should not favour any particular area on the "equatorial plane" of this coordinate system; in other words, the isomorphism from one copy of the fundamental domain to another applies equally well everywhere within the fundamental domain - it is a Clifford transformation. (This is by assumption - "generator pairs", or "type II pairs", only give spikes in PSHs if Clifford transformations are present.)

The spatial distribution of pairs in the BoPs responsible for the two peaks in Fig. 4 around $7000 h^{-1} \mathrm{Mpc}$ are shown in Fig. 6. Clearly, most of these nearly parallel pair vectors come from a selection effect in the compilation of catalogues we used. The plots for the other high spikes in Fig. 4 are very similar.

As described in Sect. 2.2.3, this type of selection effect should be removable by applying the $L_{\text {selec }}$ criterion. We tried several values for this parameter, and found that the $L_{\text {selec }}$ criterion works effectively for values $L_{\text {selec }} \geq 150 \mathrm{~h}^{-1} \mathrm{Mpc}$. By applying $L_{\text {selec }}=150 h^{-1} \mathrm{Mpc}$, the "clumped" pairs in the BoPs represented in Fig. 4 disappear and, as a result, the $L_{\text {selec }}$-filtered BoPs contain much less pairs. The histogram is now the one shown in Fig. 7; clearly the BoPs shown in Fig. 6 no longer contain many more pairs than any other BoPs, of which most must be BoPs representing noise.

There is no longer any obviously significant signal among the remaining BoPs. The highest peak represents the most abundant BoP, with 8 pairs. Figure 8 shows the spatial distribution of the pairs for this particular BoP. The averaged direction they point in has the following galactic coordinates: $l=241.4^{\circ}, b=78.2^{\circ}$. The average length of the vectors in this BoP (i.e. the length of the putative topology generator) is $6511 h^{-1}$ Mpc.

\section{Discussion, conclusions and future work}

Several filters that remove noise from the pair separation histogram (PSH) have been presented and applied: these clearly show that the PSH method can be improved on to find weak signals and to remove false signals.

The remaining best peak in the filtered PSH, from the full AGN compilation, is somewhat similar in its low significance level to that of Weatherley et al. (2003). They repeated the search made by Fagundes \& Wichoski (1987); i.e. assuming that the Milky Way was once a quasar, they looked for its "ghost" images using up-to-date quasar catalogues including the $2 \mathrm{dF}$ Galaxy Redshift Survey (2dFGRS) ${ }^{4}$ (Colless et al. 2001, 2003) and SDSS/DR1 (Schneider et al. 2002). Out of the 7 antipodal pairs that might be potential topological images of the Galaxy, 6 involve objects belonging either to 2dFGRS or to SDSS or both. So, removal of the 2dFGRS and SDSS data from the analysis by Weatherley et al. (2003) would yield only one solution. As in our own analysis, an apparent 7-fold

4 The 2dFGRS is now integrated with the $2 \mathrm{dF}$ QSO survey (Colless et al. 2003). 

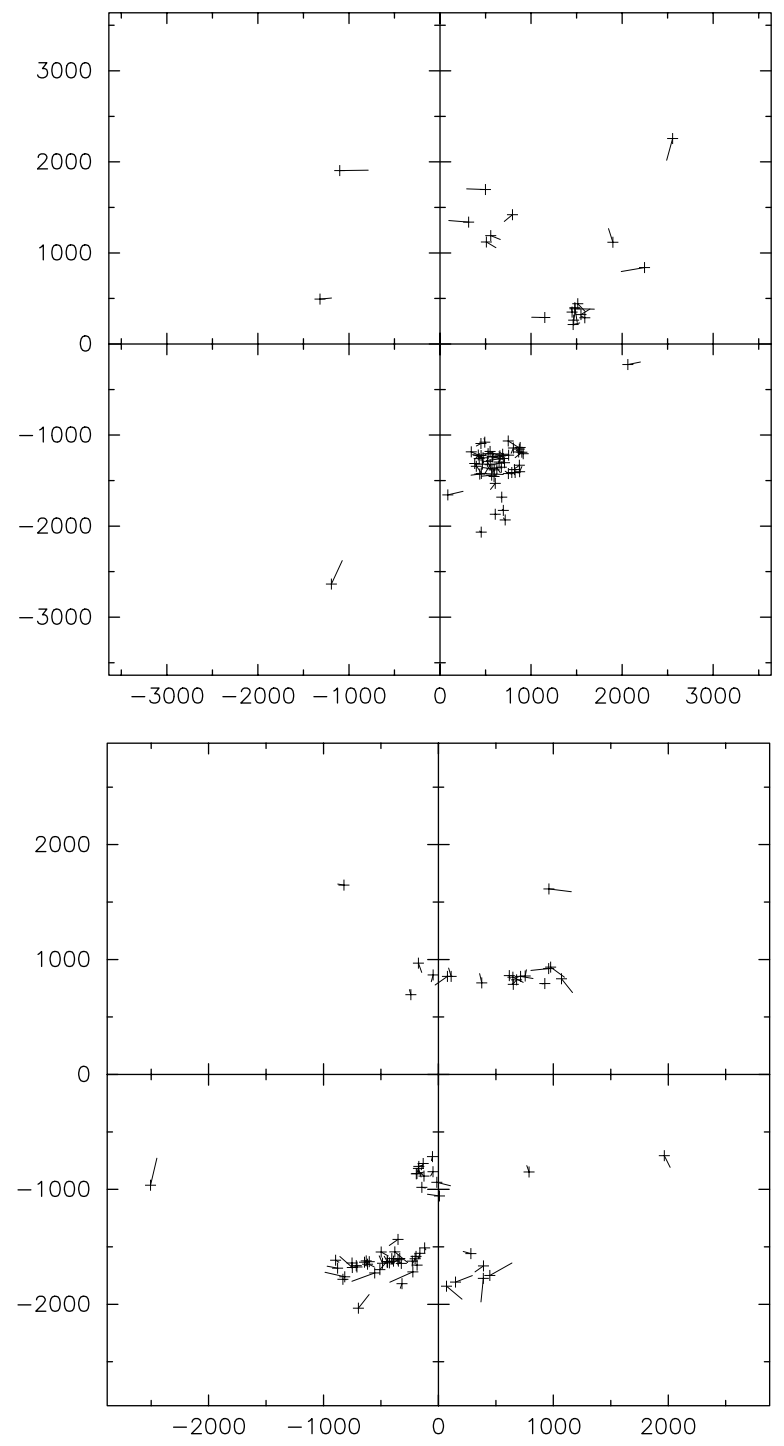

Fig. 6. Locations of pairs in the BoPs represented by the two highest peaks around $7000 h^{-1} \mathrm{Mpc}$ in Fig. 4, shown as their projections onto the equatorial plane of the cartesian coordinate system in which the direction of the $z$ axis is aligned with the direction the BoP points to. (The coordinates are in $h^{-1} \mathrm{Mpc}$.) An overwhelming majority of pairs are highly concentrated in one or two regions, which is what would be expected from selection effects which mimic a topological signal. A real topological isomorphism should generate BoPs throughout the plane, not just in a few regions.

increase is capable of mimicking a strong topological signal; the authors conclude that their result shows no significant difference in the number of pairs found over the number expected purely by chance.

It seems there are two ways to correct for this inhomogeneity. The first one is obvious: one has to use a comprehensive, large area (whole sky, at best) catalogue obtained via a uniform procedure. The SDSS, when completed, will be a very good resource for tackling this problem, even though it will only cover $\pi$ steradians $^{5}$.

\footnotetext{
${ }^{5}$ See http://www.sdss. org for details.
}

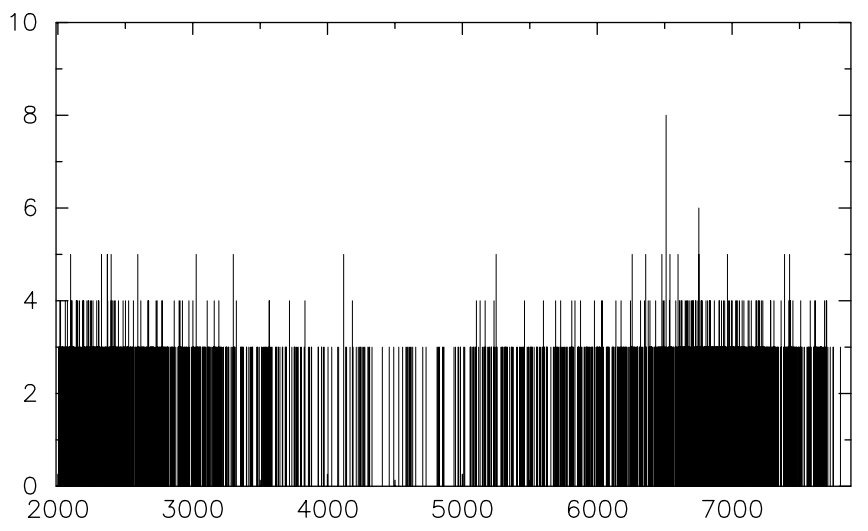

Fig. 7. PSH from Fig. 4 with the $L_{\text {selec }}=150 h^{-1} \mathrm{Mpc}$ criterion imposed. The horizontal axis shows separations of objects in pairs in $h^{-1} \mathrm{Mpc}$. The vertical axis shows the number of nearly parallel pairs in a bunch. As in the case shown in Figs. 3 and 4, we allowed for 0.5\% difference of redshifts in each pair; and as in Fig. 4, the minimum angular separation of pairs in a bunch is $\Delta \theta<0.075 \mathrm{rad}$ with a minimum of $n_{\text {pairs }}=3$ pairs. Clearly, the $L_{\text {selec }}$ criterion removes most selection effect BoPs.

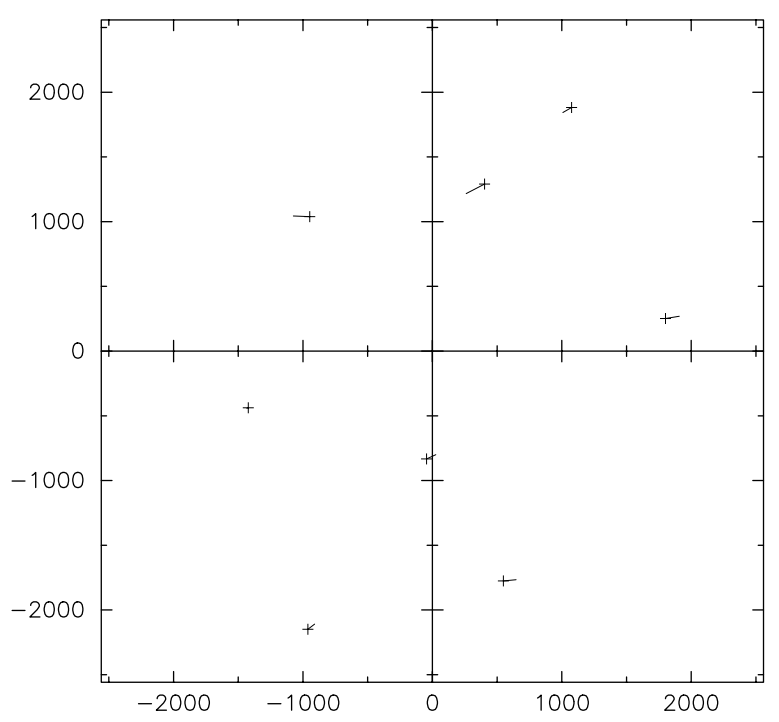

Fig. 8. Locations of pairs in the BoP represented as the highest peak in Fig. 7 shown in a cartesian coordinate system analogous to that in Fig. 6. Thanks to the $L_{\text {selec }}$ criterion, there are no selection-effectgenerated clumps of pairs. The remaining pairs are either the tail of a distribution of BoPs satisfying all the filters; or they could possibly be a topological signal.

Note, however, that the early SDSS releases, especially SDSS/DR1 (Schneider et al. 2002) ${ }^{6}$ have an intrinsic problem that outweighs any of the normal observational problems such as apparent magnitude incompleteness. Since these observations are carried out in relatively narrow strips $\left(\sim 5-10^{\circ}\right)$, of which two of the main ones lie along opposite sides of the celestial equator - and one lies fairly near the celestial equator a large fraction of the quasars in the catalogue necessarily lie in what is nearly a single plane. So, many separation vectors will necessarily be nearly parallel and have nearly identical lengths, because that is the geometry of the catalogue.

\footnotetext{
${ }^{6}$ http://www.sdss.org/dr1/
} 
As the SDSS increases to cover a large solid angle, this problem will become less important but will perhaps persist. Thus, the filters presented in this paper, $\Delta \theta$ in Sect. 2.2.2 and $L_{\text {selec }}$ in Sect. 2.2.3, still will be needed. They could potentially be "tuned" to the specific geometry of the SDSS in order to filter out the expected "selection effect" spikes caused by the survey geometry. Additional filters with spatial orientations specifically designed for the celestial positions of the SDSS selection function, in contrast to the generic filters presented here, could also help remove false signals.

It is interesting to note at this point that the SDSS has its radio counterpart: the VLA Faint Images of the Radio Sky at Twenty-cm survey (FIRST, White et al. 1997); and this FIRST catalogue might be used instead of SDSS. The radio oriented approach has an important virtue that might help not only to detect a possible topological signal in the future when the complete SDSS data becomes available, but also help to further test a candidate generator and to choose the right topological model, if such a signal exists. Instead of considering AGNs as mere point-like objects, as has always been done so far, one can explore their morphologies. The FIRST catalogue contains 811000 sources, which implies that at the apparent magnitude $m(v) \sim 24$ limit of SDSS, $\sim 50 \%$ of the optical counterparts to FIRST sources will be detected.

The availability of the maps (two-dimensional images) of radio objects listed in the FIRST catalogue provides a completely new possibility to design a statistical test, such as the PSH method combined with the filters presented above, by comparison of the morphologies of potential pairs of topologically lensed radio-loud AGNs (RLAGNs).

We do realise that the power of this method is limited by a crucial factor: radio structures of AGNs are known to evolve rather quickly. This requires fine accuracy in the redshift data. When fully available, the SDSS, with an accuracy of radial velocity estimation reaching $30 \mathrm{~km} \mathrm{~s}^{-1}$, will give an opportunity to apply this method. In other words, if there is a statistically significant clue that a set of objects seen at identical redshifts could be pairs of topologically lensed images, then they should exhibit a substantial degree of morphological similarity, after correction for the projection effects caused by different viewing angles.

These projection effects imply another caveat: the beaming effect changes the appearance of RLAGNs considerably, so the search for topologically lensed images has to be confined to objects in nearly antipodal positions within a pair. If that constraint is pushed even further, i.e. to (almost) exact antipodal positions, then this method becomes restricted to a special case, i.e. to the approach explored by Fagundes \& Wichoski (1987) and followed-up by Weatherley et al. (2003), and provides yet another chance to check if we can see topological ghosts of the Milky Way. The difference between the two methods in that case is that for an RLAGN pair at a redshift $z$ corresponding to distance $L$ from the observer, Fagundes \& Wichoski (1987) and Weatherley et al. (2003) only consider generators of length $L$, while the more general PSH method, constrained to (almost) exact antipodal positions, considers both generators of length $L$ and of length $2 L$.
Our results can be summarised as follows:

(1) Application of the $\Delta z / z=0.005$ filter (Sects. 2.2.1 and 3.2.1) to a catalogue of 15762 objects with $z>0.1$ from an observational compilation of AGN catalogues enables removal of most of the roughly $10^{8}$ pairs from the pair separation histogram (PSH), which for physical reasons the short lifetimes of AGNs - are much more likely to be noise than pairs satisfying the filter. This is shown in Fig. 3.

(2) Application of the $\Delta \theta=0.075 \mathrm{rad}$ and $n_{\text {pairs }}=3$ filters, which select out only those bunches of pairs (BoPs) which are consistent with being caused by an isomorphism (generator) between copies of the fundamental polyhedron (Sects. 2.2.2 and 3.2.2), and thereby further removes noise from the PSH, results in further improvement in the signalto-noise, and yields many BoPs which become obviously visible - see Fig. 4.

(3) However, these BoPs are best interpreted as selection effect spikes (Sect. 2.2.3). A length criterion $L_{\text {selec }} 150 h^{-1} \mathrm{Mpc}$ to remove those BoPs most likely to be selection effects is effective (Sect. 3.2.3) in doing so.

(4) The highest spike remaining after these filters (Fig. 7), points to the galactic direction $\left(l=241.4^{\circ}, b=78.2^{\circ}\right)$ and is of length $6511 \mathrm{~h}^{-1} \mathrm{Mpc}$. However, it is not highly significant.

When the full SDSS becomes available, applying this method would definitely be worth trying in order to extract any signal that might be present. Even though WMAP analyses have so far indicated that no topological signal is likely to be present at scales much below the horizon scale, it would be imprudent to rely on one observational method alone to consider such a fundamental cosmological property - the shape of the Universe - to be unmeasurable on these scales.

Another prospect for future work is that if an interesting signal were found, then comparing radio maps of the putative topologically lensed RLAGNs would seem to be a promising tool for discriminating between true and false topological signals.

Acknowledgements. S.B. acknowledges support from KBN Grant 1P03D 01226.

\section{References}

Colless, M., Dalton, G., Maddox, S., et al. 2001, MNRAS, 328, 1039

Colless, M., Peterson, B. A., Jackson, C., et al. 2003

[arXiv: astro-ph/0306581]

Cornish, N. J., Spergel, D. N., Starkman, G. D., \& Komatsu, E. 2004, Phys. Rev. Lett., 92, 201302

de Sitter, W. 1917, MNRAS, 78, 3

Dowker, F., \& Surya, S. 1998, Phys. Rev. D, 58, 124019

Fagundes, H. V., \& Gausmann, E. 1999, Phys. Lett. A, 261, 235

Fagundes, H. V., \& Wichoski, U. F. 1987, ApJ, 322, L5

Flesch, E., \& Hardcastle, M. J. 2004, A\&A, 427, 387

Friedman, A. 1923, Mir kak prostranstvo i vremya (The Universe as Space and Time), Leningrad, Akademya

Friedman, A. 1924, Z. Phys., 21, 326

Gomero, G. I., Teixeira, A. F. F., Rebouças, M. J., \& Bernui, A. 2002, Int. J. Mod. Phys. D, 11, 869

Hatziminaoglou, E., Siemiginowska, A., \& Elvis, M. 2001, ApJ, 547, 90 
Hawking, S. W. 1984a, Nucl. Phys. B, 244, 135

Hawking, S. W. 1984b, Nucl. Phys. B, 239, 257

Lachièze-Rey, M., \& Luminet, J. 1995, Phys. Rep., 254, 135

Lehoucq, R., Lachièze-Rey, M., \& Luminet, J.-P. 1996, A\&A, 313, 339

Luminet, J., \& Roukema, B. F. 1999, in NATO ASIC Proc., 541, Theoretical and Observational Cosmology, 117 [arXiv: astro-ph/9901364]

Luminet, J., Weeks, J. R., Riazuelo, A., Lehoucq, R., \& Uzan, J. 2003, Nature, 425, 593

Naselsky, P. D., Doroshkevich, A. G., \& Verkhodanov, O. V. 2003, ApJ, 599, L53

Naselsky, P. D., Doroshkevich, A. G., \& Verkhodanov, O. V. 2004, MNRAS, 349, 695

Rebouças, M. J., \& Gomero, G. I. 2004, Braz. J. Phys., 34, 1358

Roukema, B. F. 1996, MNRAS, 283, 1147

Roukema, B. F. 2002a, Adv. Space Res., 31, 449

Roukema, B. F. 2002b, in Marcel Grossmann IX Conference on General Relativity, ed. V. G. Gurzadyan, R. T. Jantzen, \& R. Ruffini (Singapore: World Scientific), 1937
Roukema, B. F., Lew, B., Cechowska, M., Marecki, A., \& Bajtlik, S. 2004, A\&A, 423, 821

Schneider, D. P., Richards, G. T., Fan, X., et al. 2002, AJ, 123, 567

Schwarzschild, K. 1900, Vier. Astr. Gess., 35, 337

Schwarzschild, K. 1998, Class. Quant. Grav., 15, 2539

Sievers, J. L., Bond, J. R., Cartwright, J. K., et al. 2003, ApJ, 591, 599

Spergel, D. N., Verde, L., Peiris, H. V., et al. 2003, ApJS, 148, 175

Uzan, J.-P., Lehoucq, R., \& Luminet, J.-P. 1999a, in Proc. of the XIX ${ }^{\text {th }}$ Texas meeting, Paris 14-18 December 1998, ed. E. Aubourg, T. Montmerle, J. Paul, \& P. Peter, article $N^{\circ}$ 04/25 [arXiv:gr-qc/0005128]

Uzan, J.-P., Lehoucq, R., \& Luminet, J.-P. 1999b, A\&A, 351, 766

Weatherley, S. J., Warren, S. J., Croom, S. M., et al. 2003, MNRAS, $342,9 \mathrm{~L}$

Weeks, J., Lehoucq, R., \& Uzan, J. 2003, Class. Quant. Grav., 20, 1529

White, R. L., Becker, R. H., Helfand, D. J., \& Gregg, M. D. 1997, ApJ, 475, 479

Zeldovich, I. B., \& Grishchuk, L. P. 1984, MNRAS, 207, 23 